\title{
Pure virtual braids homotopic to the identity braid
}

by

\author{
H. A. Dye (Lebanon, IL)
}

\begin{abstract}
Two virtual link diagrams are homotopic if one may be transformed into the other by a sequence of virtual Reidemeister moves, classical Reidemeister moves, and self crossing changes. We recall the pure virtual braid group. We then describe the set of pure virtual braids that are homotopic to the identity braid.
\end{abstract}

1. Introduction. A virtual link diagram is a decorated immersion of $n$ copies of $S^{1}$ with two types of crossings: classical and virtual. Classical crossings are indicated by over/under markings and virtual crossings are indicated by a solid encircled $\mathrm{X}$. An example of a virtual link diagram is shown in Figure 1.

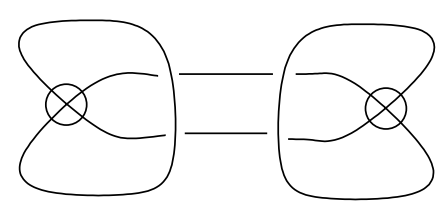

Fig. 1. Kishino's knot

Virtual link theory is a generalization of classical knot theory that was introduced by Louis H. Kauffman in 1996 (see [9]). Two virtual link diagrams are said to be equivalent if one may be transformed into the other by a sequence of classical Reidemeister moves (shown in Figure 2) and virtual Reidemeister moves (shown in Figure 3). Classical link diagrams contain no virtual crossings and form a subset of the virtual link diagrams.
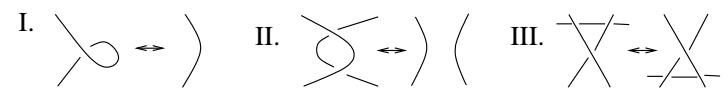

Fig. 2. Classical Reidemeister moves

2000 Mathematics Subject Classification: Primary 57M27.

Key words and phrases: virtual braids, link homotopy, identity braid. 
I.

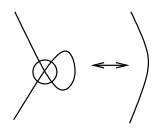

II.

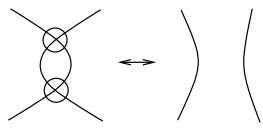

III.

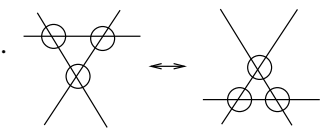

IV.

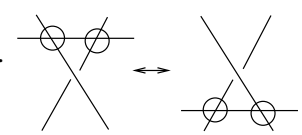

Fig. 3. Virtual Reidemeister moves

A representation of a virtual link diagram is a pair $(F, L)$ where $L$ is a link diagram on a closed, two-dimensional surface $F$ taken up to Dehn twists and handle $\left(S^{1} \times I\right)$ additions and cancellations. Reidemeister moves can be performed on the surface.

REMARK 1.1. Detailed descriptions of representations are given in [3] or [9]. Abstract surfaces (punctured representations) are described in [7].

We recall the following theorems:

Theorem 1.1 (See [7] and [9]). Classes of representations are in oneto-one correspondence with equivalence classes of virtual link diagrams.

TheOREM 1.2 (Kuperberg [11]). Representations of virtual link diagrams have a unique representative embedding class in the minimal genus surface that can support the diagram.

Recalling [4], two virtual link diagrams are defined to be homotopic if one diagram may be transformed into the other by a sequence of virtual Reidemeister moves, classical Reidemeister moves, and self crossing changes. (By self crossing change, we mean changing the over/under markings at a crossing between two segments of the same link component.)

In this paper, we focus on virtual braids (see [8] and [10]) and pure virtual braids. A $n$-strand virtual braid diagram is a decorated immersion of $n$ copies of $[0,1]$ into the plane. Let $\left\{f_{1}, \ldots, f_{n}\right\}$ denote the $n$ components. The sets of endpoints $\left\{f_{1}(i), \ldots, f_{n}(i)\right\}, i \in\{0,1\}$, are contained in a line for each $i$. We refer to the set of points where $i=0$ as the upper endpoints and the other set $(i=1)$ as the lower endpoints, as shown in Figure 4. Two virtual braid diagrams are said to be virtually homotopic if one can be transformed into the other by a sequence of Reidemeister moves, virtual Reidemeister moves, and self crossing changes that leave the endpoints fixed. A virtual braid is pure if the braid is equivalent to a braid with no self crossings, that is, every crossing involves two distinct components. For a pure virtual braid, the order of the set of upper endpoints is the same as the order of the set of lower endpoints. We denote the set of $n$-strand pure virtual braids as $\mathrm{VP}_{n}$ 


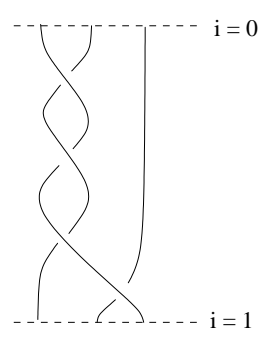

Fig. 4. Example of a braid diagram

and the set of $n$-strand pure classical braids as $\mathrm{BP}_{n}$. We will discuss the group structure of $\mathrm{VP}_{n}$ and $\mathrm{BP}_{n}$ in the next section.

Two virtual braid diagrams are virtually homotopic if one diagram can be transformed into the other by a sequence of Reidemeister moves, virtual Reidemeister moves and self crossing changes. We will denote the set of homotopic $n$-strand pure virtual braids as $H\left(\mathrm{VP}_{n}\right)$ and the set of homotopic $n$-strand pure classical braids as $H\left(\mathrm{BP}_{n}\right)$ following the notation in [5].

A representation of an $n$-strand pure virtual braid is a pair $(D, b)$ where $D$ is a once punctured, two-dimensional oriented surface with an immersed braid $b$ (where the boundary points of $b$ are contained in the boundary of $D)$ modulo Reidemeister moves, Dehn twists, and handle cancellations and additions.

REMARK 1.2. We can view the surface $D$ as $I \times I$ with $m$ attached handles $(m \geq 0)$. Theorems 1.1 and 1.2 apply to representations of pure virtual braids. In a diagram of a representation with genus one, we will draw only the handle as shown in Figure 5. Elements of $\mathrm{VP}_{n}$ with genus one representations have a natural correspondence with elements of $\mathrm{BP}_{n+2}$ and $\mathrm{BP}_{n+1}$.

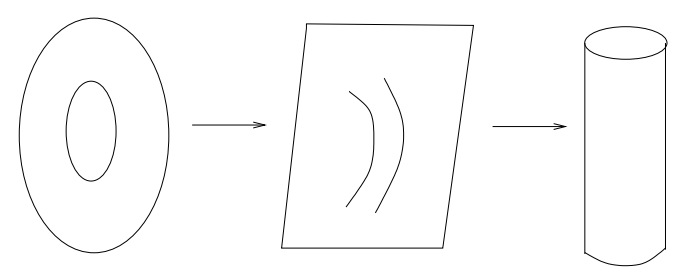

Fig. 5. Surfaces for genus one representations

Classical homotopy has been studied by Milnor [12], Goldsmith [5], and more recently Habegger and Lin [6]. Significant differences exist between the classical case and the virtual case. Strikingly, not all virtual knot diagrams are homotopic to the unknot. (This topic has been explored in [4] where Milnor's link groups and $\mu$-invariants are applied to virtual link diagrams.) 
The difference in the case of braids is illustrated by the fact that every classical braid with a fixed ordering on the endpoints can be homotoped into a braid with no self crossings. This is not true in the virtual case, as shown in Figure 6. In this paper, we determine which pure virtual braids are homotopic to the identity braid.
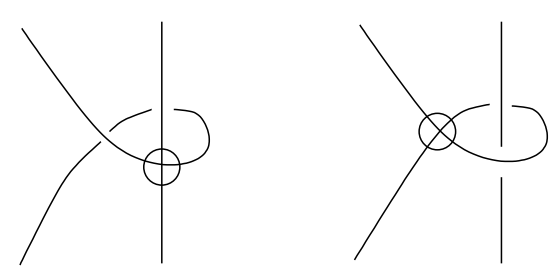

Fig. 6. Braids not homotopic to a pure braid

2. Pure braids. The structure of the $n$-strand pure virtual braid group is described in [2]. $\mathrm{VP}_{n}$ is generated by the set $\left\{\lambda_{i k}, \lambda_{k i} \mid i, k \in\{1, \ldots, n\}\right\}$. These generators are illustrated in Figure 7.
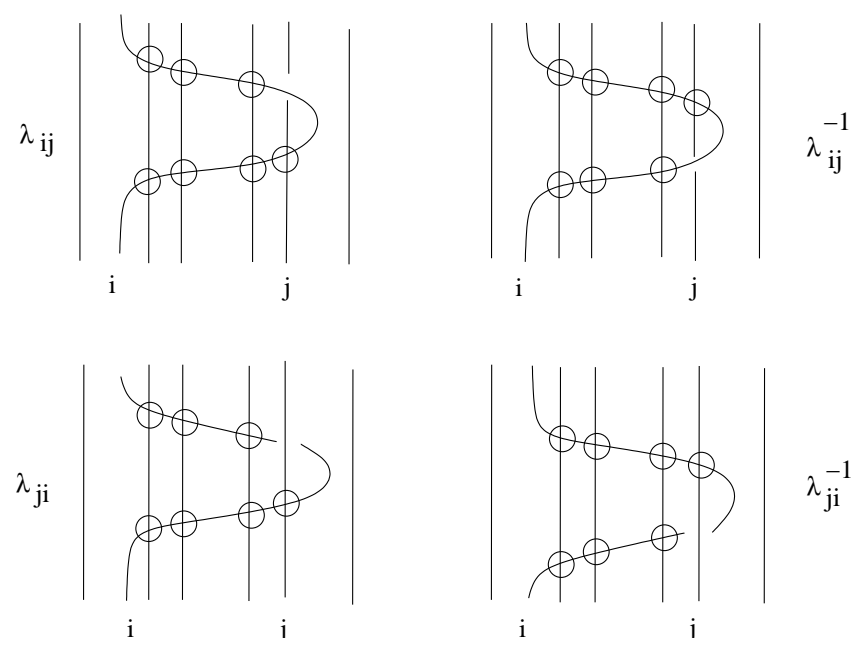

Fig. 7. Generators of the pure virtual braid group

The relations in $\mathrm{VP}_{n}$ are given in equations (1) and (2) below (cf. [2]). First,

$$
\lambda_{j k} \lambda_{i n}=\lambda_{i n} \lambda_{j k} \quad \text { for distinct } i, j, k, n .
$$

Let $s(i j)=1$ if $i<j$ and -1 otherwise. Then

$$
\lambda_{k i}^{s(k i)} \lambda_{k j}^{s(k j)} \lambda_{i j}^{s(i j)}=\lambda_{i j}^{s(i j)} \lambda_{k j}^{s(i j)} \lambda_{k i}^{s(k i)} .
$$


REMARK 2.1. The naming convention for the generators differs slightly from that given in [2]. In this paper, the two indices indicate the strands involved. The classical crossing is in the upper tier of crossings. The first index number indicates the over crossing strand while the second indicates the under crossing strand.

The $n$-strand pure virtual braids have a group structure where multiplication is performed by concatenating braids as shown in Figure 8.

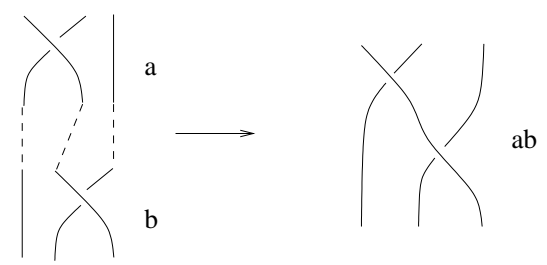

Fig. 8. Braid multiplication

The $n$-strand pure classical braids, denoted $\mathrm{BP}_{n}$, form a subgroup of $\mathrm{VP}_{n}$. The generators of $\mathrm{BP}_{n}$ are denoted as $\sigma_{i j}$ with $1 \leq i<j \leq n$. Each $\sigma_{i j}$ can be expressed as a product of the virtual generators:

$$
\sigma_{i j}=\left(\lambda_{i, i+1} \lambda_{i, i+2} \ldots \lambda_{i, j-1}\right)\left(\lambda_{i j} \lambda_{j i}^{-1}\right)\left(\lambda_{i, j-1}^{-1} \ldots \lambda_{i, i+2}^{-1} \lambda_{i, i-1}^{-1}\right) .
$$

Recall the commutator of two elements:

$$
[x, y]=x y x^{-1} y^{-1} .
$$

Let $F_{i}$ denote the subgroup of $\mathrm{BP}_{n}$ generated by $\left\{\sigma_{i, i+1}, \sigma_{i, i+2}, \ldots, \sigma_{i n}\right\}$. The set of $n$-strand pure classical braids homotopic to the identity is the smallest normal subgroup generated by the commutators

$$
\left[\sigma_{i j}, g \sigma_{i j} g^{-1}\right] \quad \text { where } g \in F_{i} .
$$

From [2], we can describe $\mathrm{VP}_{n}$ as a semidirect product and give a normal form for pure virtual braids. Let $V_{n}$ denote the set of generators $\left\{\lambda_{i n}^{ \pm 1}, \lambda_{n i}^{ \pm 1} \mid\right.$ $i \in 1, \ldots, n-1\}$. Then $V_{n}^{*}$ denotes the smallest normal subgroup generated by $V_{n}$ in VP. The subgroup $V_{n}^{*}$ is normal in $\mathrm{VP}_{n}$, and $\mathrm{VP}_{n}$ is the semidirect product $V_{n}^{*} \rtimes \mathrm{VP}_{n-1}$. That is, if $w_{n} \in V_{n}^{*}$ then

$$
w_{n}=\prod_{j=1}^{k} g_{j} a_{j} g_{j}^{-1} \quad \text { where } a_{j} \in V_{n}, g_{j} \in \mathrm{VP}_{n-1} .
$$

Based on the normal form, we define the length of $w_{n}$ to be $k$. The braid $w_{n}$ has minimal homotopic length if every strand contains at least one real crossing and the braid does not contain a subsequence such that its deletion produces a braid which homotopic to $w_{n}$ but not virtually equivalent. 
REMARK 2.2. If a braid has a strand with only virtual crossings then this braid is equivalent to a conjugate of a braid with only classical crossings on this strand. However, the minimal length of the braid (based on the normal form) increases as shown in Figure 9.

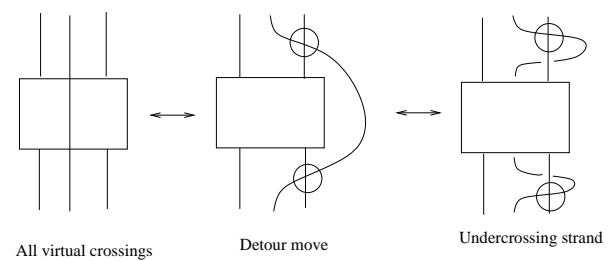

Fig. 9. Converting to a braid with minimal homotopic length

The following theorem describes a normal form for elements of $\mathrm{VP}_{n}$.

TheOREM 2.1 (see [2]). Let $b$ be an element of $\mathrm{VP}_{n}$. Then $b=w_{2} w_{3} \ldots w_{n}$ where $w_{j} \in V_{j}^{*}$.

REMARK 2.3. There is a reduced form for $g_{j}$ when describing elements of $V_{n}^{*}$ based on the relations given earlier.

This result parallels Artin's theorem [1] about the normal form of pure classical braids. In the next section, we describe the set of pure virtual braids that are homotopic to the identity braid. We incorporate Goldsmith's methodology from the classical case [5] and representations of virtual braids [3].

3. Genus and homotopy. We prove that the set of $n$-strand pure braids homotopic to the identity braid is a normal subgroup of $\mathrm{VP}_{n}$. We will denote this subgroup as $I\left(\mathrm{VP}_{n}\right)$. To describe it, we will first prove a sequence of lemmas about minimal genus and $V_{n}^{*}$. We then apply this result to a braid in normal form in the next section.

Lemma 3.1. Let $w \in \mathrm{VP}_{2}$. Then a minimal genus representation of $w$ has genus less than or equal to one.

Proof. The braid $w$ contains two strands. Immerse the second strand in a surface with one handle so that the second strand follows the longitude of the handle as shown in Figure 10.

COROLlary 3.2. The elements of $\mathrm{VP}_{2}$ can be viewed as elements of $\mathrm{BP}_{3}$ and there are non-classical elements of $\mathrm{VP}_{2}$ homotopic to the identity braid.

Proof. If $w$ is an element of $\mathrm{VP}_{2}$ then $w$ has a genus one representation. This representation can be viewed as an element of $\mathrm{BP}_{3}$. There are nontrivial braids in $\mathrm{BP}_{3}$ that are homotopic to the identity braid. These braids 

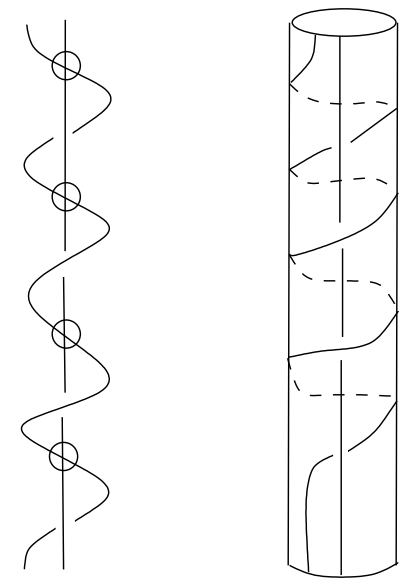

Fig. 10. A representation of an element of $\mathrm{VP}_{2}$

in $\mathrm{BP}_{3}$ correspond to representations of non-trivial elements of $\mathrm{VP}_{2}$, which are then homotopic to the identity braid.

We apply the following lemma in the cases where $n \geq 3$.

LEMMA 3.3. Let $h$ be a handle in a minimal genus representation of a pure virtual braid. Let $\left\{s_{1}, \ldots, s_{k}\right\}$ be the collection of strands such that the removal of $s_{i}$ admits a cancellation curve. Then $k \leq 2$.

Proof. Let $[m]$ denote the meridian and $[l]$ the longitude of handle $h$. Suppose that removing strand $s_{1}$ from the representation admits a cancellation curve $\alpha_{1}$ on handle $h$. Then the strand $s_{1}$ intersects the cancellation curve $\alpha_{1}$ transversely. By hypothesis, removing strand $s_{2}$ admits a cancellation curve $\alpha_{2}$ on $h$. Note that $s_{2}$ intersects $\alpha_{2}$ transversely.

Suppose that $\alpha_{2}$ is homotopic to $\alpha_{1}$; then $s_{1}$ also intersects both $\alpha_{1}$ and $\alpha_{2}$ transversely. As a result, $\alpha_{2}$ cannot be a cancellation curve after the removal of $s_{2}$.

We observe that $\alpha_{1}$ and $\alpha_{2}$ are not homotopic but both pass through handle $h$. Assume without loss of generality that $\alpha_{1}=[\mathrm{m}]$ and $\alpha_{2}=[l]$ (implying that $s_{1}$ parallels the longitude and $s_{2}$ parallels the meridian in the handle). Let $s_{3}$ be a third strand in the braid whose removal admits a canceling curve $\alpha_{3}$ for $h$. The curve $\alpha_{3}$ is homotopic to neither curve. But if $\alpha_{3}$ passes through $h$ then $\alpha_{3}$ is homotopic to a curve that wraps around the meridian $a$ times and the longitude $b$ times. Hence, $\alpha_{3}$ intersects at least one of the pair, $\alpha_{1}$ or $\alpha_{2}$. But then at least one of the pair, $s_{1}$ or $s_{2}$, intersects $\alpha_{3}$ transversely. Hence, $\alpha_{3}$ cannot be a cancellation curve.

Lemma 3.3 can be applied to show that certain elements of $V_{n}^{*}$ with $n \geq 4$ have representations with minimal genus less than or equal to one. 
Lemma 3.4. Let $n \geq 4$. If $w$ is a non-classical element of $V_{n}^{*}$ with minimal homotopic length then the minimal genus of a representation of $w$ is one.

Proof. Let $w$ be an element of $V_{n}^{*}$ that is homotopic to the identity such that every strand contains at least one real crossing. Suppose that a minimal genus surface for $w$ has $m$ handles. If strand $s_{n}$ is removed then each handle admits a canceling curve since removing $s_{n}$ results in the identity braid. If strand $s_{i}$ is removed then $m-1$ handles admit a canceling curve. As a result, if some handle admits only two canceling curves then all other curves admit three or more canceling curves since $n \geq 4$. Now, by Lemma 3.3 there is at most one handle and the representation has genus less than or equal to one.

The remaining case occurs when $n=3$. We will need the following lemma about linking number. Recall that the sign of a classical crossing, $c$, is determined by its relative orientation as shown in Figure 11.

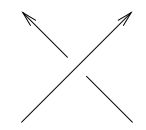

$+1$

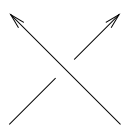

$-1$

Fig. 11. Sign of a classical crossing

Let $V$ be the collection of crossings where strand $a$ passes over strand $b$. Then

$$
\operatorname{Link}(a, b)=\sum_{c \in V} \operatorname{sgn}(c) .
$$

Proposition 3.5. Let braid $b \in \mathrm{VP}_{n}$ be homotopic to the $n$-strand identity braid. If $b$ contains $n$ copies of the generator $\lambda_{i j}$, then $b$ contains $n$ copies of the generator $\lambda_{i j}^{-1}$.

Proof. For the generator $\lambda_{i j}, \operatorname{Link}(i, j)=-1$ and $\operatorname{Link}(j, i)=1$. Linking number is a homotopy invariant and in the identity braid $\operatorname{Link}(i, j)=0$. Hence the generator $\lambda_{i j}$ is paired with the generator $\lambda_{i j}^{-1}$. lemma.

Let $\lambda_{p(i j)}$ represent either $\lambda_{i j}$ or $\lambda_{j i}$. We use this notation in the following

Lemma 3.6. If $w$ is a braid in $V_{3}^{*}$ with minimal homotopic length that is homotopic to the identity then the minimal genus of a representation of $w$ is less than or equal to one.

Proof. Let $w$ be a braid in $V_{3}^{*}$ with minimal homotopic length that is homotopic to the identity braid. Suppose that a minimal genus representation of $w$ is a surface with $m$ handles. Consider the representation of $w$. Since 
$w$ is in normal form, the removal of the 3rd strand from the representation result in the 2 -strand identity braid and $m$ canceling curves on the surface.

The removal of the first strand results in the braid $x$, a 2-strand braid that is homotopic to the identity, and as a result, $m-1$ canceling curves on the surface. Similarly, the removal of the 2nd strand results in the braid $y$, a 2-strand braid homotopic to the identity and $m-1$ canceling curves on the surface.

Applying Lemma 3.3, we observe that the representation of $w$ contained at most two handles. Note that if $x$ is classical, then $x$ is the identity braid and the representation of $w$ has genus less than or equal to one. As a result, we will assume that both $x$ and $y$ are non-classical for the remainder of the proof.

Let $\widehat{x}$ denote the subsequence of $w$ consisting of conjugates of $\lambda_{p(13)}^{ \pm 1}$. The removal of the second strand from $\widehat{x}$ results in a 2-strand braid homotopic to the identity, while the removal of the 1st and 3rd strands results in the identity braid. Hence, a minimal genus representation of $\widehat{x}$ has genus one. (Note that if the genus is zero, then $\widehat{x}$ is a classical braid, which contradicts our assumption that $x$ is a non-classical braid.) Let $\widehat{y}$ denote the subsequence of $w$ consisting of conjugates of $\lambda_{p(23)}^{ \pm 1}$. Following the argument given for $\widehat{x}$, a minimal genus representation of $\widehat{y}$ also has genus one.

Now, $w=x_{1} y_{1} x_{2} y_{2} \ldots x_{n} y_{n}$ where $\widehat{x}=x_{1} x_{2} \ldots x_{n}$ and $\widehat{y}=y_{1} y_{2} \ldots y_{n}$. Because minimal genus representations of $\widehat{x}$ and $\widehat{y}$ have genus one, we may assume that (after isotopy) minimal genus representations of each subbraid $x_{i}$ and $y_{i}$ occur on a tube (possibly) with handles as shown in Figure 12.

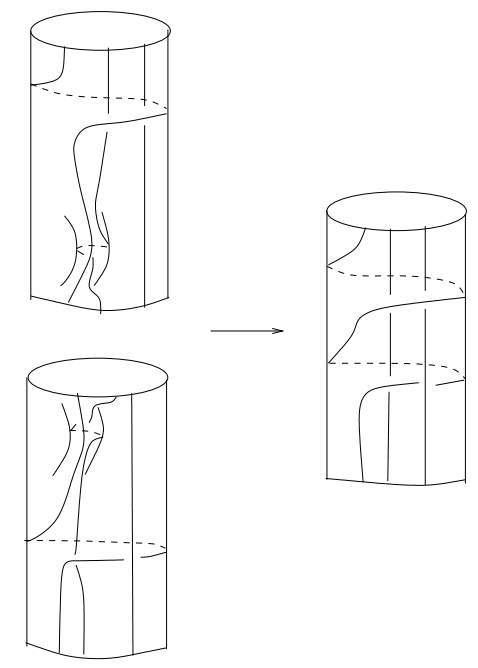

Fig. 12. Minimal genus surface 
However, a representation of $w$ has at most genus two. If handles occur in a representation of some $x_{i}$ (or $y_{i}$ ) then all the handles are canceled in a representation of $\widehat{x}$ (or $\widehat{y}$ ). Without loss of generality, assume that the tube representation of some $x_{i}$ contains at least one handle. (The handles can be selected so that a handle either contains strands 1 and 3 or strands 1 and 2.) Suppose that this handle contains strands 1 and 3 ; then the subbraid $x_{i+1}$ must have a corresponding handle involving these strands.

In the braid $w$, the braid $y_{i}$ occurs between $x_{i}$ and $x_{i+1}$. As a result, the handle in $x_{i}$ cancels with a handle occurring in either $y_{i-1}$ or $y_{i}$. But $\widehat{y}$ contains no virtual or classical crossings between 1 and 3 . Hence, the handles cannot be canceled in $w$ and the minimal genus of $w$ is three, a contradiction.

Suppose that the handles in $x_{i}$ that cannot be removed in $w$ contain strands 1 and 2 . That is, $g_{i}^{-1}$ does not cancel with $g_{i+1}$. Now, the deletion of either strand 1 or strand 2 admits a canceling curve for each of these handles, but not the tube. The deletion of strand 3 admits a canceling curve for every handle. Again by applying Lemma 3.3, we observe that the representation of $w$ has genus one.

We have proved the following theorem:

TheOREM 3.7. Let $w_{n}$ be a minimal length, non-trivial element of $V_{n}^{*}$ such that every strand contains a real crossing. If $w_{n}$ is homotopic to the identity braid then a minimal genus representation of $w_{n}$ has genus less than or equal to one.

REMARK 3.1. If a braid contains a strand with only virtual crossings then some representation contains a handle with this strand immersed along the longitude.

LEMMA 3.8. Let $b=w_{2} w_{3} \ldots w_{n}$ be an element of $\mathrm{VP}_{n}$ (where $w_{j} \in$ $\left.V_{j}^{*}\right)$. Then $b$ is homotopic to the $n$-strand identity braid if and only if each $w_{i}$ is homotopic to the identity braid.

Proof. If each $w_{i}$ is an element of $I\left(\mathrm{VP}_{n}\right)$ then $b=w_{1} w_{2} \ldots w_{n}$ is an element of $I\left(\mathrm{VP}_{n}\right)$. Let $w$ be an element of $I\left(\mathrm{VP}_{n}\right)$. Then there is a homotopy sequence $\left\{p_{0}, p_{1}, \ldots, p_{m}\right\}$, with $w=p_{0}$ and $p_{m}$ equivalent to the identity braid, that transforms $w$ into the identity. Remove strand $n$ from each diagram and replace it with the identity strand. This reduces $w$ to the braid $b_{n-1}$ where $b_{n-1}=w_{1} \ldots w_{n-1}$. This preserves the homotopy sequence so that $b_{n-1}$ is homotopic to the identity strand. Removing strands, we observe that $b_{i}=w_{1} \ldots w_{i}$ is homotopic to the identity braid. Hence, each $w_{i}$ is homotopic to the identity braid.

4. Braids homotopic to the identity braid. We show that the set of braids homotopic to the identity braid forms a normal subgroup of $\mathrm{VP}_{n}$. Let 
$x$ be an element of $\mathrm{VP}_{n}$ with $x=\left(\lambda_{i-1, i} \lambda_{i-2, i} \ldots \lambda_{1 i}\right)\left(\lambda_{n i}^{-1}\right)\left(\lambda_{n-1, i}^{-1} \ldots \lambda_{i+1, i}^{-1}\right)$. Let $g_{a}$ and $g_{b}$ denote classical braids generated by the set

$$
\left\{\sigma_{1 i}, \sigma_{2 i} \ldots \sigma_{i-1, i} \sigma_{i, i+1} \ldots \sigma_{i n}\right\} \text {. }
$$

Proposition 4.1. If an element of $\mathrm{VP}_{n}$ has the form

$$
\left[\sigma_{i j}, g_{a} x g_{b} \sigma_{i j} g_{b}^{-1} x^{-1} g_{a}^{-1}\right]
$$

(as shown below in Figure 17 where $g_{a}$ and $g_{b}$ are denoted as $A$ and $B$ ) then the braid is homotopic to the identity braid.

Proof. We show sample homotopy sequences for elements of $\mathrm{VP}_{2}$ and $\mathrm{VP}_{3}$ in Figures 13 and 14.

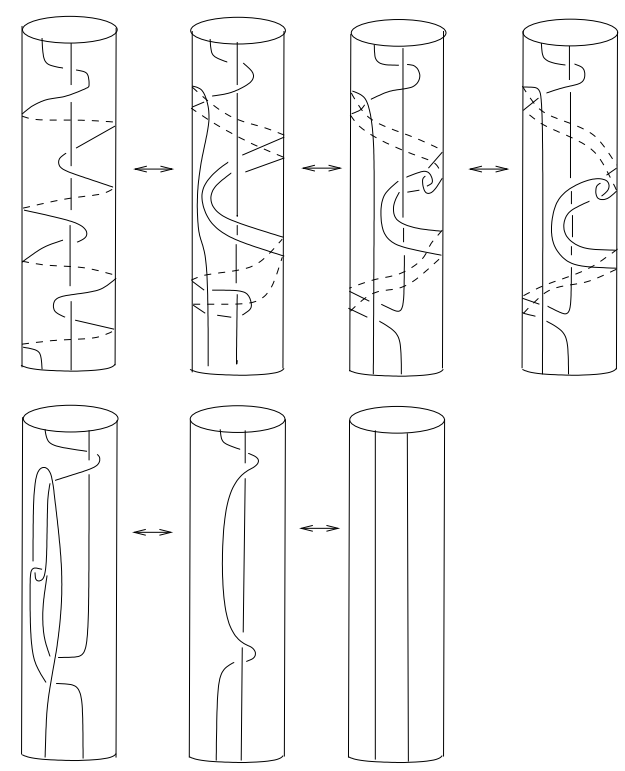

Fig. 13. A homotopy sequence in $\mathrm{VP}_{2}$

REMARK 4.1. This braid can be expressed as the product of an element of $V_{n}^{*}$ and $\mathrm{VP}_{n-1}$.

Proposition 4.2. If $w$ is an element of $V_{n}^{*}$ that is homotopic to the identity with minimal homotopic length then some representation of $w$ corresponds to an element of $\mathrm{BP}_{n+1}$ of the form $\left[\sigma_{i j}, g \sigma_{i j} g^{-1}\right]$.

Proof. Let $w$ be a braid in $V_{n}^{*}$ with minimal homotopic length that is homotopic to the identity. By Theorem 3.7, the braid $w$ has a representation with minimal genus one, a torus. View the torus as the complement of two linked curves to obtain an element of $\mathrm{BP}_{n+2}$. After removing strand 


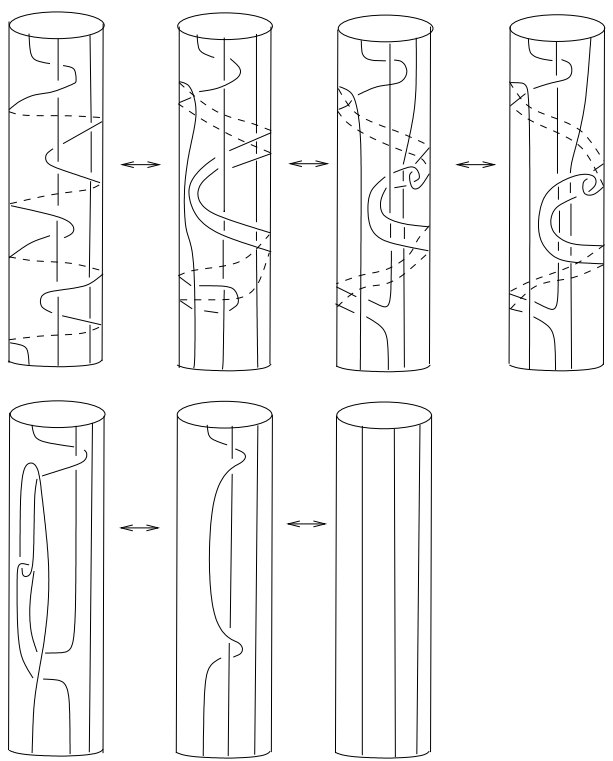

Fig. 14. A homotopy sequence in $\mathrm{VP}_{3}$

$n+2$, we obtain a classical braid in $\mathrm{BP}_{n+1}$ that is homotopic to the identity. The braid $w$, as an element of $\mathrm{BP}_{n+1}$, can be written in the classical normal form, $b_{1} b_{2} \ldots b_{n+1}$, where $b_{i}$ is an element of the group generated by $\left\{\sigma_{i, i+1}, \sigma_{i, i+2}, \ldots, \sigma_{i, n+1}\right\}$. In $\mathrm{BP}_{n+1}$, a braid homotopic to the identity has the form $\left[\sigma_{i j}, g \sigma_{i j} g^{-1}\right]$.

In a representation of $w$ the longitude of the torus corresponds to strand $n+1$. As a result, consider the first $b_{i}$ that includes strand $n+1$.

Now, $\sigma_{i, n+1}$ either holds the place of $\sigma_{i j}$ or is a term in $g$ from equation (3). Let $x$ denote the element of $\mathrm{VP}_{n}$ shown in Figure 15.

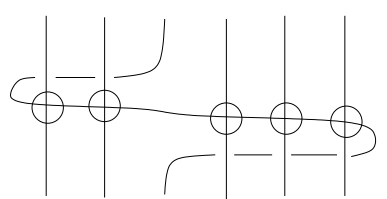

Fig. 15. Braid $x$ in $\mathrm{VP}_{n}$

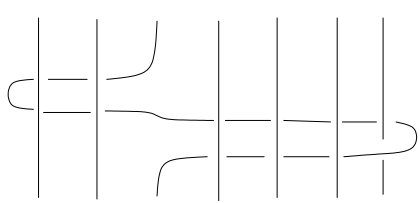

Fig. 16. Braid $\widehat{x}$ in $\mathrm{BP}_{n+1}$

In $\mathrm{VP}_{n}$,

$$
x=\left(\lambda_{i-1, i} \lambda_{i-2, i} \ldots \lambda_{1 i}\right)\left(\lambda_{n i}^{-1} \lambda_{n-1, i}^{-1} \ldots \lambda_{i+1, i}^{-1}\right) .
$$

From a representation of $x$, we obtain $\widehat{x}$, the element of $\mathrm{BP}_{n+1}$ shown in Figure 16. In $\mathrm{BP}_{n+1}$,

$$
\widehat{x}=\left(\sigma_{i, i+1}^{-1} \sigma_{i, i+2}^{-1} \ldots \sigma_{i, n}^{-1}\right) \sigma_{i, n+1}^{-1}\left(\sigma_{i n} \ldots \sigma_{i, i+1}\right) .
$$

If $\sigma_{i, n+1}$ corresponds to $\sigma_{i j}$ then the homotopy sequence requires a crossing change on strand $n+1$. Hence $g$ must contain the term $\sigma_{i n+1}$ and the braid 
$b_{i}$ has the form

$$
\left[\sigma_{i j}, q_{a} \widehat{x} q_{b} \sigma_{i j} q_{b}^{-1} \widehat{x}^{-1} q_{a}^{-1}\right]
$$

where $q_{a}$ and $q_{b}$ are classical braids in the group generated by $\left\{\sigma_{i, i+1} \ldots \sigma_{i n}\right\}$. This corresponds to the braid shown in Figure 17. In terms of $\mathrm{VP}_{n}$ this is a braid of the form

$$
\left[\sigma_{i j}, G_{a} \lambda_{n i}^{-1} G_{b} \sigma_{i j} G_{b}^{-1} \lambda_{n i} G_{a}^{-1}\right]
$$
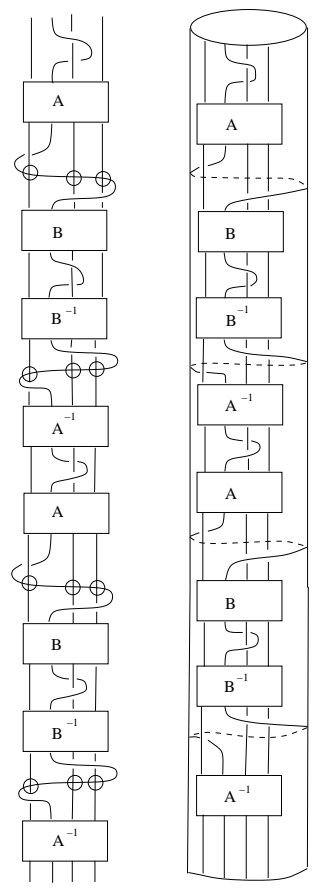

Fig. 17. A representation of $w$

(Note that $G_{a}$ and $G_{b}$ are the images of $q_{a}$ and $q_{b}$ with the appropriate part of $\widehat{x}$ in $\mathrm{VP}_{n}$.) Let

$$
\begin{aligned}
\lambda & =\lambda_{n i}, \\
z & =G_{a}^{-1} \sigma_{i j} G_{a}, \\
y & =G_{b} \sigma_{i j} G_{b} .
\end{aligned}
$$

Then the braid in equation (4) can be rewritten as

$$
G_{a}\left(z \lambda^{-1} y \lambda z^{-1} \lambda^{-1} y^{-1} \lambda\right) G_{a}^{-1} .
$$

This can be rewritten in normal form for $\mathrm{VP}_{n}$ :

$$
G_{a}\left(z y z^{-1} y^{-1}\right)\left(y z y^{-1} \lambda^{-1} y z^{-1} y^{-1}\right)\left(y z \lambda z^{-1} y^{-1}\right)\left(y \lambda^{-1} y^{-1}\right) \lambda G_{a}^{-1} .
$$

The braid $z y z^{-1} y^{-1}$ is an element of $\mathrm{VP}_{n-1}$ and homotopic to the identity. Then $y z y^{-1}$ is homotopic to the braid $z$. Canceling terms, we obtain the 
braid

$$
G_{a} z \lambda^{-1} y \lambda z^{-1} \lambda^{-1} y^{-1} \lambda G_{a}^{-1} .
$$

Using equations (5)-(7) to rewrite, we obtain

$$
\sigma_{i j} G_{a} \lambda^{-1} G_{b} \sigma_{i j} G_{b}^{-1} \lambda G_{a}^{-1} \sigma_{i j} G_{a} \lambda^{-1} G_{b} \sigma_{i j} G_{b}^{-1} \lambda G_{a}^{-1} .
$$

This is the same form as the original braid.

REMARK 4.2. Note that in this form, strand $i$ underpasses all $i-1$ previous strands. Any weaving on these strands can be described by multiplying $w$ by the braid $b_{k}$ with $k \leq i$. Note that if the original braid $w$ over crosses the $i-1$ previous strands, then $b_{k}$ with $k<i$ involves crossings on strand $n+1$. This contradicts the fact that $b_{i}$ is the first strand to have crossings involving strand $n+1$.

We have proved the following theorem:

THEOREM 4.3. The set of pure virtual braids homotopic to the identity is the smallest normal subgroup generated by elements of the form

$$
\left[\sigma_{i j}, g_{a} x g_{b} \sigma_{i j} g_{b}^{-1} x^{-1} g_{a}^{-1}\right]
$$

where $x=\left(\lambda_{i-1, i} \lambda_{i-2, i} \ldots \lambda_{1 i}\right)\left(\lambda_{n i}^{-1} \lambda_{n-1, i}^{-1} \ldots \lambda_{i+1, i}^{-1}\right)$ or $x$ is the identity braid and $g_{a}, g_{b}$ are classical braids generated by the set $\left\{\sigma_{1 i}, \sigma_{2 i}, \ldots \sigma_{i-1, i}\right.$, $\left.\sigma_{i, i+1} \ldots \sigma_{i n}\right\}$.

Acknowledgments. This research was performed while the author held a National Research Council Research Associateship Award jointly at the Army Research Laboratory and the U.S. Military Academy. This effort was sponsored in part by the Office of the Dean at the United States Military Academy. The U.S. Government is authorized to reproduce and distribute preprints for Government purposes notwithstanding any copyright annotations thereon. The views and conclusions contained herein are those of the author and should not be interpreted as necessarily representing the official policies or endorsements, either expressed or implied, of the United States Military Academy or the U.S. Government. (Copyright 2008.)

\section{References}

[1] E. Artin, Theory of braids, Ann. of Math. (2) 48 (1947), 101-126.

[2] V. G. Bardakov, The virtual and universal braids, Fund. Math. 184 (2004), 1-18.

[3] H. A. Dye and L. H. Kauffman, Minimal surface representations of virtual knots and links, Algebr. Geom. Topology 5 (2005), 509-535.

[4] —, 一, Virtual homotopy, preprint, arXiv:math/0611076.

[5] D. L. Goldsmith, Homotopy of braids - in answer to a question of E. Artin, in: Topology Conference (Blacksburg, VA, 1973), Lecture Notes in Math. 375, Springer, Berlin, 1974, 91-96. 
[6] N. Habegger and X.-S. Lin, The classification of links up to link-homotopy, J. Amer. Math. Soc. 3 (1990), 389-419.

[7] N. Kamada and S. Kamada, Abstract link diagrams and virtual knots, J. Knot Theory Ramif. 9 (2000), 93-106.

[8] S. Kamada, Invariants of virtual braids and a remark on left stabilizations and virtual exchange moves, Kobe J. Math. 21 (2004), 33-49.

[9] L. H. Kauffman, Virtual knot theory, Eur. J. Combin. 20 (1999), 663-690.

[10] L. H. Kauffman and S. Lambropoulou, Virtual braids, Fund. Math. 184 (2004), 159-186.

[11] G. Kuperberg, What is a virtual link?, Algebr. Geom. Topology 3 (2003), 587-591.

[12] J. Milnor, Link groups, Ann. of Math. (2) 59 (1954), 177-195.

McKendree University

701 College Road

Lebanon, IL 62254, U.S.A.

E-mail: hadye@mckendree.edu

Received 7 June 2007;

in revised form 21 August 2008 